\title{
Product design exploration based on users psychological perception
}

\author{
Yue Zhang ${ }^{1, a}$, Yuan Ren ${ }^{2, b}$ \\ 1,2Jingdezhen University, Art department, Jingdezhen 333001 \\ a,b414708353@qq.com
}

Keywords: User psychology; Product design; Sensorial perception

Abstract. When choosing product, those visual, auditory, tactile and other sensorial perceptions are the key factors deciding whether to buy it. And the product's function, shape, color and surface treatment is the key points that considered when conceiving. Through exploration on users psychological and spiritual demand during the using process, this paper emphasizes the physical and functional form of product, focusing on user's psychological perception experience in order to create outstanding products that in line with the users psychological and physiological needs.

\section{Users psychological perception}

Users psychological perception is a kind of psychological judgment about product shape, quality, color, function and other factors produced through the user's heart, which based on consumer's awareness of experience and determines the individual mental behavior. Sensing image refers the association on product by their own senses of product, it is related to visual factor, user living experience, cultural background, etc., and these belongs to the user psychological period ${ }^{[1]}$. Users psychology shows personal performance, but also have similarities. Individual users have different consumption concept and mind because of different nation, ethnic and different geographical areas. Gradually transit from basic survival and security to multi-consumption patterns, the people's needs not only contain physical needs, but also has to meet the spiritual pleasure. Meanwhile, the user's growth, living, studding environment and personal psychology have differences, these all resulted diversification. The user psychology keep changing by the user's age, interests, educational environment, grade and life, and all these conclude the endless pursuit.

\section{User perception in product design}

Sensory experiences produced by the five senses. The sensory appeal is to create a variety of perceptual experience, including those sensory stimulation caused by vision, hearing, touch, taste and smell, etc.. For example, in the product experience, the key factor is to increase the product sensory experience, and to promote the interaction and communication between the product and the people ${ }^{[2]}$. When purchasing and using product, through its own experience, including a variety of factors, such as perceived product ideas, spirit and will, take advantage of a variety of human sensory stimulation to perceive the product shape, color and material, allowing users to create a good impression of the product.

Visual perception product design. Vision is the main thoroughfare of people receiving outside information, and the product perception captured more from the shape, color,

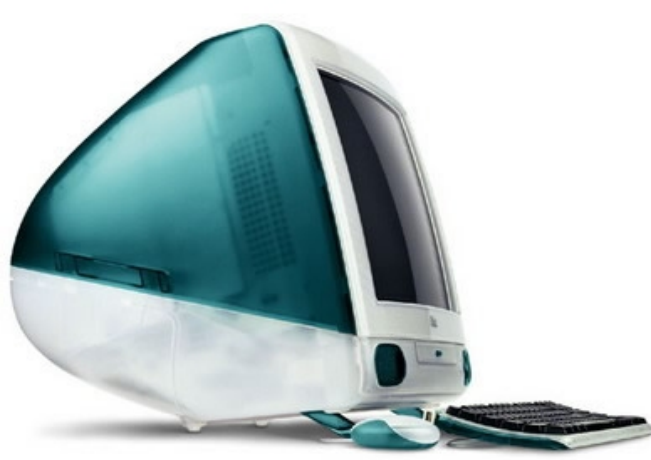

Fig.1 Apple imac series texture, size and so on. Visual perception allows people to feel the product for the first time. Such as 
the common Apple imac series (Fig. 1), firstly using transparent material shell, vaguely revealing the internal structure of the product, breaking the traditional computer in black, white and gray design based on color, and breaking the stereotype computer image, graceful curves reveal human design. The use of transparent material and bright colors give consumers a strong sense of visual impact, highly infected people's vision, and ingratiated the modern pursuit of consumption concept and psychology.

Auditory perception in product design. Auditory sense is the second largest human sensory system. Through the auditory senses, outside sound information was accepted in order to properly communicate with the outside world, work and life. The sound of the product becomes the communication medium between man and machine. Sound is an important information element of the product features indicated. Products can make people perceive normal operations by the normal machine sound. Sound can transmit a sense of security, such as the clicking sound when turning a lock, as well as refrigerators;s zoom sound, and printers' rustle sounds all convey "properly working" message.

Tactile Perception in product design. Touch is the most direct sensory system of the product for users. The product's own value information conveyed to people, especially the surface texture of the product, is particularly sensitive and advantageous to tactile perception. Such as the uneven texture of a product, give people a hard, cold, smooth, soft, and other different feelings. Compared with vision and auditory sense, touch allows directly feel on product, and can be more real, delicate, brings more real reception.

The tactile perception mainly achieved by dealing with the materials. Meanwhile, the replacement of product material is sometimes a reflection of the changing times, and also is the fashion leader. Products have multiple sensory perception intertwined, such as integration of tactile sensations in the visual design process, which is "visual-tactile." Whether in product design, or other design behavior, form design is not only arise visual experience affection, but also express some kind of touching and emotional expressions.

Other sensory perception in product design. Besides visual, auditory and touch senses, the sense of smell and taste are also important sensory organs. Smell is an organ that give people unique sense, and it also lasts for longest time. Smell mainly takes the gas as a carrier, but not all products are odor. Smell has been used in many industries, such as furniture and food. In product design, it is the most hard perception through tasting, because products can not be eaten. But the soul of design is innovation, such as using toothpick after eating, toothpick with flavors can become the expression theme of designer. In Japan, it has developed scented toothpick, lemon cups, honey-flavored straw etc., these are all fine taste product design.

Design is a process, and its contents is complex, involving various unqualified elements on psychological aspects, have great relevance with people's perception. Pay attention to people's needs are the vane of future design performance, it will drift in the future design sky.

\section{User perception and product design}

Functional design based on user perception. To achieve the functionality of a product requires functional design. It needs the designer to expand product functional analysis based on economic principles from users' potential needs, make functional definitions, do product design by professionals, enterprise for production. Through pricing analysis, conduct targeted marketing, make business out of homogeneity product trap. Functional design is essentially the theory of deepening market segmentation, which has a good variety, but in the final analysis are based on functional segmentation. 
More functions is not mean better product. For different user groups, it is necessary to carry out the appropriate changes in product function. For mature user groups, a psychologically inherent think is that product should meet the needs of users. According to the actual situation of their own needs, to choose a more practical goods. Targeted products functional design can not resulted high cost because of too many functions. Low market price will be easier to fit the consumption concept of those people. Designers need to solve question on the consumers' position from the marketing view, and fully satisfied consumers' psychological needs. When positioning functional design, all models should be properly designed and refined.

Product form design based on user perception. As the first element of product information transfer, product form enables the product intrinsic quality, organization, structure, content and other factors turn to the external image factors, and gives a physiological and psychological processes through vision, and these are closely related with feeling, composition, structure, material, color, space, and other functions ${ }^{[3]}$. Product form is the carrier and outside expression of the function. Designers often use specific modeling language for product form design, express the products' function and design concept through the outside image. Those information expressed from the product forms are also the common judgment and measure benchmark of the consumer when perceiving the product image.

In the shape design based on the user perception, the experience on product should keep certain possibilities, allowing consumers to feel the product content and emotion when using. This is a kind of enduring emotion on experiences, but not an outburst natural emotional reflection. After using, the profound experience on shape and function, and the delicate understanding ponder turn to be personal emotion. Carefully created by designers, the product shape based on users' emotional psychology can be shaped out.

Color design based on user perception. Color is the visual perception, color design is ingenious arrangement of colors. While the nature is colorful, color design is ever-changing. When observing the around environment, colors take the first break into people's eyes, producing a wide range of visual effects, bringing a different visual experience, and directly affect people's aesthetic perception, mood swings and even life state, working efficiency ${ }^{[4]}$.

Color is a subjective psychological performance. Many colors have shown a different perception of psychological characteristics. Such as red, gives a passionate, energetic, and vibrant feeling. Red has an auspicious meaning in the traditional concept of our country, representing the best of luck. But also can be reminiscent of the red blood and killing, have dangerous characteristics, giving fear, dangerous, brutal feeling, often regard the red as warning color. Besides, for different people, even the same color may arise different feeling. When color designing, the basic and social features should be considered first, as well as its psychological affection. Meanwhile, designers should understand the users favorite color, in order to create psychological suitable product for consumers.

Surface treatment based on user perception. The so-called surface treatment is to form mechanical, physical and chemical different layer on the surface of a substrate by human. Its purpose is to satisfy he corrosion resistance, abrasion resistance, decorative features, or other special requirements of the product.

Advances in technology make the production technology has been greatly developed, many of the existing market products have been created different tactile and visual effect. Although the purchase on perception is for different texture and different surface treatment, one thing is the same, that is comfort. 


\section{Conclusion}

Product design process is the realization of the product artistic value and practical value. Seize the user's consumer psychology, mix designer's creative concept with demand in order to create user-satisfied products. Designers put their experience and aesthetic accomplishment in the image of the product, let them to be verified by the market. It is not a simple burst of passion, nor a talk of design concept, but is detail design demand based on the market and users. Beside good modeling capability, consumers' psychological needs should be fully considered and be satisfied. By changing user needs to create more excellent products.

\section{References}

[1] LIN RT, LIN CY, WONG J. An application of multidimensional scaling in product semantics[J] International Journal of Industrial Ergonomics, 1996, 18 (2): 193-204.

[2] Qun Huang. Exploration on emotional experience and perception characteristics of product[J]national trade economic core journals 2008 (32): 279.

[3] Yanli Su. Product form design [M]. Shanghai Science and Technology Press. 2010,01.

[4] Chengyuan Ren. Color design [M]. Machinery Industry Press .20109,03. 